\title{
Beam width assessment of a Linear Array for MaMIMO applications at 3.5 GHz using measurements and raytracing
}

\author{
Maarten Velghe ${ }^{1}$, Sergei Shikhantsov ${ }^{1}$, Luc Martens ${ }^{1}$, Wout Joseph ${ }^{1}$ and Arno Thielens ${ }^{1}$
}

(1) Department of Information Technology, Ghent University/IMEC, Technologiepark 126, 9052 Ghent, Belgium

\begin{abstract}
The width of a beam produced by MaMIMO arrays will affect a user's exposure to RF-EMFs. We performed measurements in an anechoic chamber using a virtual arrays and successfully assessed this beamwidth. We validated our measurements with simulations.

\section{Introduction}

In the fifth generation of telecommunication networks, Massive Multiple-input-multiple-output (MaMIMO, [1]) base stations (BSs) will produce narrow RF-EMF beams aimed at each specific user device they service. Knowledge on the widths of these beams is essential to evaluate a user's exposure to RF-EMFs. The aim of this study is to assess this beam width via measurements in an anechoic chamber and to validate the used setup with free-space simulations.
\end{abstract}

\section{Materials and Method}

\subsection{Measurement setup}

Figure 1 shows a schematic top view of the measurement setup in the anechoic chamber. Two vertically polarized dipole dual cone broadband antennas, a transmitting (TX) and receiving $(\mathrm{RX})$ antenna, are connected to a vector network analyzer (VNA) performing measurements at 3.5 GHz. The TX antenna is fixed on a linear positioning system, moving along the y-axis. The RX antenna is placed on a $2 \mathrm{D}$ positioning system, consisting of two orthogonally oriented linear positioners moving along the $y$ - and $x$-axis. Positioning systems are co-planar, such that the antennas stay in the same xy-plane as they move.

The TX grid has $17 \times 1$ locations, with the interspacing chosen to be $\delta \mathrm{Tx}=4.28 \mathrm{~cm}$, which is about half the wavelength. This results in an array aperture $\mathrm{L}$ of $68 \mathrm{~cm}$. The TX-RX distance D is chosen to be $68 \mathrm{~cm}$ as well. The RX grid has 33x17 locations (33 elements along the $y-$ axis per 17 elements along the $\mathrm{X}$-axis) with an interspacing half of the TX interspacing $(\delta \mathrm{Rx}=2.14 \mathrm{~cm})$. We measure the channel transfer function $h_{k n}$, measured between each Tx-position $\mathrm{k}(\mathrm{k}=1 \ldots 17)$ and each $\mathrm{Rx}-$ position $\mathrm{n}(\mathrm{n}=1 \ldots 561)$, resulting in the channel matrix $\mathbf{H}_{\text {measured. }}$

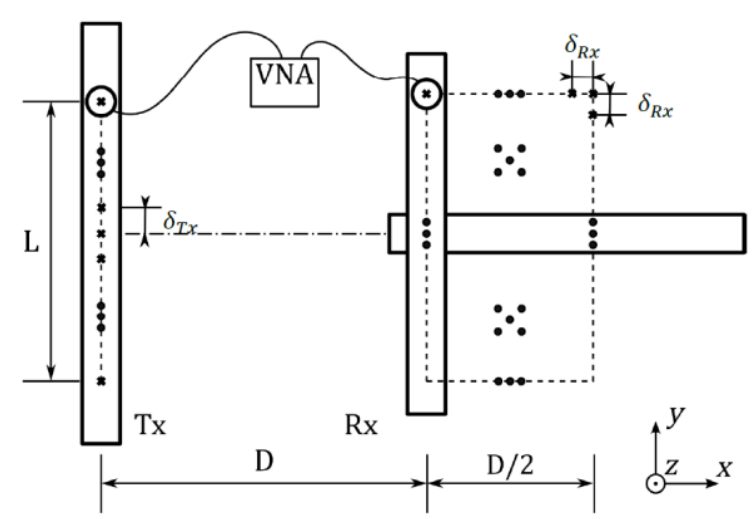

Figure 1: Schematic overview of the measurement setup.

To validate the measurement results, we estimate the wireless channel between the TX and RX virtual arrays using a Line-of-Sight (LOS) propagation model. This is suitable for calculating propagation in the anechoic chamber with virtual arrays, as it only takes into account direct propagation paths between TX-RX pairs and neglects mutual coupling effects of the arrays' antennas. This results in the simulated channel matrix $\mathbf{H}_{\text {model }}$.

\subsection{Post processing}

The channel correlation matrix $(\mathrm{CM})$ is commonly used for the analysis of the performance of MaMIMO systems is defined as:

$$
G=H^{*} H \text {. }
$$

This results in two $561 \times 561$ CMs: Gmeasured and $\mathbf{G}_{\text {model }}$ which are complex valued with real values on the main diagonal. To simplify the analysis we take the average of the results in each of the $17 \mathrm{Rx}$-rows along the $\mathrm{x}$-axis. This way we calculate the average beam width over the distance $x=[68 \mathrm{~cm} 102 \mathrm{~cm}]$. This results in the $33 \times 33$ averaged CMs $\mathbf{G}_{\text {avg,measured }}$ and $\mathbf{G}_{\text {avg,model. The }}$ are normalized.

To assess the beamwidth, we define the spatial correlation function (CF) $\rho\left(G_{-}\right.$avg,i) as the average over the ith diagonal of $\mathbf{G}_{\text {avg: }}$ :

$$
\rho\left(\mathbf{G}_{\text {avg }}, \mathrm{i}\right)=\frac{\sum_{\mathrm{k}=1}^{33-\mathrm{i}}\left|\mathrm{g}_{\mathrm{k}, \mathrm{k}+\mathrm{i}}\right|}{33-\mathrm{i}}
$$

with glm an element of $\mathbf{G}_{\text {avg. }} \rho$ can be treated as a function of the distance in the y-direction between the receivers. 
The average relative difference $\sigma_{\text {avg }}$ between $\mathbf{G}_{\text {avg,measured }}$ and $\mathbf{G}_{\text {avg,model is calculated as: }}$

$$
\sigma_{\text {avg,lm }}=\left|\frac{\left|g_{\text {avg,model,lm }}\right|-\left|g_{\text {avg,measured,lm }}\right|}{\left|\frac{\left|g_{\text {avg,model,lm }}\right|+\left|g_{\text {avg,measured,lm }}\right|}{2}\right|}\right|
$$

with $\sigma_{\mathrm{avg}, \operatorname{lm}}$ an element of $\sigma_{\mathrm{avg}}$.

\section{Results and Discussion}

Figure 2 compares $\rho\left(\mathbf{G}_{\text {avg,model }}\right)$ and $\rho\left(\mathbf{G}_{\text {avg,measured }}\right)$. A very good agreement is observed. Both functions have maximum at $\delta y=0$ and decrease rapidly within a 1wavelength distance $(8.57 \mathrm{~cm})$. After minor oscillations they flatten-out at around $4 \%$ of their maximum value for $\delta y>0.5 \mathrm{~m}\left(\approx 6^{*} \lambda\right)$. The distance between the maximum and half the maximum $\delta_{\mathrm{hmy}}=6.5 \mathrm{~cm}$, the beam width is thus $2 * \delta_{\mathrm{hm} y}=13 \mathrm{~cm}$.

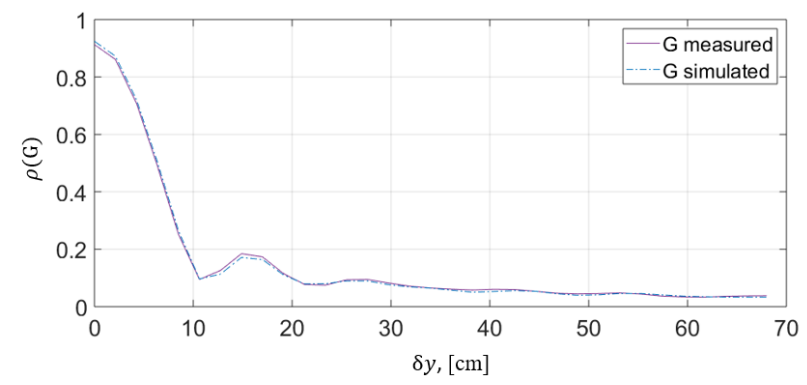

Figure 2: Spatial correlation function of $G_{\text {avg,measured }}$ and $G_{\text {avg,model }}$ in terms of the distance between their rows.

In Figure 3 the normalized $\mathrm{CMs}_{\mathrm{avg} \text {,measured }}(3 \mathrm{a}), \mathrm{G}_{\mathrm{avg} \text {,model }}$ (3b) and their difference $\sigma_{\text {avg }}$ (3c) are shown. The main diagonal dominance is apparent in both averaged CMs. The same result has been obtained in measurement campaigns [2] and using geometry-based models [3]. ravg, does not exceed $5 \%$ on the main diagonal. This implies a good agreement between measurements and simulations. However, some of the out-of-diagonal elements exceed $30 \%$. The reason for that are the low absolute correlation values observed at large RX separation distances, which are shown in the top-right corner of the CMs. Even a small variation of the received signal (due to e.g. reflections by the positioners and support structures, alignment errors, radiation pattern variation) results in a relatively high simulation error.

This measurement setup can now be used to evaluate exposure from MaMIMO systems in other environments, such as a room without absorbing materials, with obstructed line-of-sight conditions, etc.

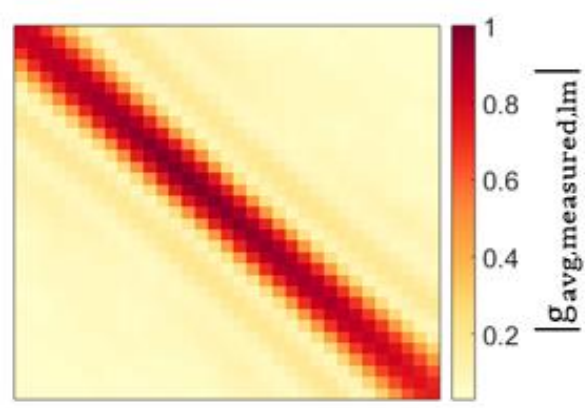

(a)

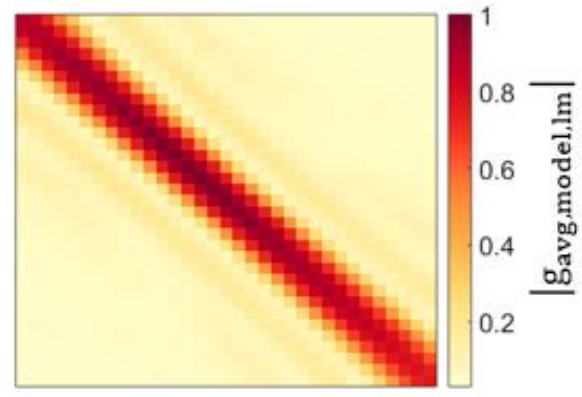

(b)

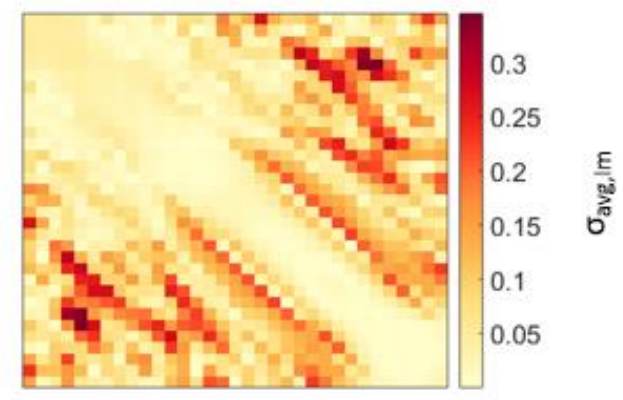

(c)

Figure 3: The normalized correlation matrices $G_{\text {avg,measured }}$ $(a), G_{\text {avg,model }}(b)$, and their relative difference $\sigma_{\text {avg }}(c)$.

\section{Conclusions}

We measured and simulated the beam width of a MaMIMO array and found $2 * \delta_{\mathrm{hm}} \mathrm{y}=13 \mathrm{~cm}$. A good agreement between measurements and simulations was observed.

\section{Acknowledgements}

This project has received funding from the European Union's Horizon 2020 research and innovation programme under the Marie Skłodowska-Curie grant agreement No 665501 with the research Foundation Flanders (FWO). A.T. is an FWO [PEGASUS]2 Marie Skłodowska-Curie Fellow

\section{References}

1. Marzetta, et al. 2010. IEEE Trans. Wirel. Commun., 9(11): 3590 .

2. Claessens, et al. 2018. IEEE International Workshop on SPAWC, 19: 1-5.

3. Marzetta, 2016. Fundamentals of Massive MIMO. Cambridge University Press 